\title{
On the Interpretation of Serial Laboratory Measurements in Acute Myocardial Infarction
}

\author{
Adelin Albert, ${ }^{1}$ Eugene K. Harris, ${ }^{2}$ Jean-Paul Chapelle, ${ }^{1}$ Camille Heusghem, ${ }^{1}$ and Henrl E. Kulbertus ${ }^{3}$
}

Serial laboratory determinations are now routinely performed on patients admitted to intensive-care units. Adequate interpretation of such cumulative information for clinical decisionmaking purposes is a challenging problem. We describe a statistical method for predicting-sequentially as the data become available - the patient's outcome, death or survival. Thus the method goes beyond previously reported techniques that base such prediction on only a single multivariate observation. The method has been applied to daily measurements of serum urea and lactate dehydrogenase, performed during one week on patients hospitalized in the coronarycare unit with acute myocardial infarction. Two baseline variables were also included in the dynamic risk index so derived: the age of the patient and the number of previous myocardial infarctions recorded on admission. We also discuss the problems of selecting the most-predictive laboratory tests and of determining for each test the amount of past data needed to achieve satisfactory prediction. We distinguish between global evaluation of the dynamic risk index obtained (in terms of specificity and sensitivity) and individual interpretation (in terms of posterior/prior probability ratio) of a given risk score for a particular patient. The approach described may contribute to more effective use of results of repeated laboratory tests on critically ill patients.

Additional Keyphrases: best use of clinical laboratory data prognostic value - myocardial infarction - statistics - urea - lactate dehydrogenase - dynamic risk index - logistic model - outcome prediction - time-dependent observations - response curves

Clinical chemistry has always played a major role in intensive-care medicine, but the many technological advances have not always been matched by comparable progress in interpretation of laboratory data.

Thus, when a patient is admitted to a coronary-care unit with suspected myocardial infarction, he is submitted to a panel of clinical laboratory tests that are systematically repeated throughout hospitalization. This yields a huge amount of information that needs to be integrated and interpreted by the clinician for diagnostic, prognostic, and decision-making purposes. Among the various laboratory tests performed, some are likely to present repetitious information. As to those tests that truly reflect the patient's status, it is questionable whether the serial measurements recorded are used optimally for outcome prediction. In this context, we propose to address the problem of continuous risk assessment in cases of myocardial infarction (MI) from cumulative laboratory data collected during the patient's

\footnotetext{
${ }^{1}$ Laboratory of Clinical Chemistry, and ${ }^{3}$ Department of Cardiology, University of Liège, 1, rue des Bonnes-Villes, 4020 Liège, Belgium.

2 Laboratory of Applied Studies, National Institutes of Health, Bethesda, MD 20205; present address, P.O. Box 710, Madison, VA 22727.

Received May 13, 1983; accepted September 26, 1983.
}

first week in the coronary-care unit. ${ }^{4}$ This problem can be investigated by biokinetic modeling techniques, as described by Groth and de Verdier (1), to derive values of quantities more closely describing the temporal changes of the pathological process. The approach developed here, however, is entirely statistical.

Improved use of serial laboratory measurements has long been claimed and recognized, but surprisingly little has been done in this area. Moreover, the role of laboratory tests in acute MI has been mainly directed toward diagnosis (2) rather than prognosis. In most papers dealing with prognostic indices, MI patients are classified into two groups, survivors and nonsurvivors, on the basis of a single multivariate observation, by well-known discriminant analysis techniques $(3,4)$. In proceeding that way, however, nonsurvivors are all grouped together and the elapsed time between admission and death is not taken into account. Further, the method provides only a static risk index and does not account for the dynamics of the disease process and possible late re-infarctions. Some authors (5) have proposed fitting a polynomial function to the series of observations and allocating the patients on the basis of the resulting coefficients, but the method is cumbersome and hardly works dynamically.

In a recent paper (6), we looked at serial determinations as forming a response curve to the disease process by linear interpolation between successive measurements of either tissue markers (CK, LD) or biochemical components (AGP, urea) that are increased by metabolic disturbances. We conjectured that the shape of the response curves of these various substances might bring valuable information for outcome prediction. These considerations led one of us to develop a theory of discriminant analysis (7) based on multivariate response curves, which was successfully applied to a retrospective data base of more than 300 patients with MI (6). Although exhibiting interesting dynamic characteristics, the method was essentially descriptive and did not account for the actual time of death of nonsurvivors, a drawback that we have already mentioned for the classical approach. This prompted us to propose a new methodology for upgrading the interpretation of serial laboratory measurements recorded from patients admitted to coronary-care units.

\section{The Data Base}

Our study material consisted of 330 patients with documented MI admitted to the two coronary-care units of the University Hospital, Liège, Belgium. Acute MI was diagnosed on the basis of typical clinical history, electrocardiographic evidence, and a rise and fall of CK and LD activities in serum.

Age (in years) and the number of previous MIs were recorded for each patient at admission. The mean age of the

\footnotetext{
4 Nonstandard abbreviations: AGP, $\alpha_{1}$-acid glycoprotein; CK, creatine kinase (EC 2.7.3.2); LD, lactate dehydrogenase (EC 1.1.1.27); MI, myocardial infarction; AIC, Akaike's information criterion; ML, maximum likelihood.
} 
patients was 57.4 (SD 9.5) years. Fifty-nine (17.9\%) of the patients were women, and $65(19.7 \%)$ had a prior history of myocardial infarction.

Blood was sampled at the time of hospital admission, and then daily until the end of the first week of hospitalization. Total CK and LD activities were measured by optimized spectrophotometric methods (CK and LD UV tests, no. 3388 and 3399; Merck, Darmstadt, F.R.G.) $(8,9)$ at $37^{\circ} \mathrm{C}$, with an ABA-100 discrete analyzer (Abbott Labs., North Chicago, IL 60064). We determined haptoglobin and AGP concentrations by standard radial immunodiffusion (10), using Partigen Plates (Behring Institute, F.R.G.). Serum urea, creatinine, and uric acid were assayed by the diacetyl monoxime (11), modified Jaffé reaction (12), and phosphotungstate (13) techniques, respectively, in a continuous-flow analyzer (SMA 12/60; Technicon Instruments Corp., Tarrytown, NY 10591).

The clinical significance and the evolutionary characteristics of these laboratory tests after an acute MI have been extensively studied and reported in a series of papers (1416). For illustrative purposes, we have restricted our attention here to the statistical treatment of three constituents only: AGP, a "positive" acute-phase reactant possibly related to the healing of the jeopardized myocardium (17); urea, which is likely to reflect proteolysis and possible hemodynamic impairment consequent to $\mathrm{MI}$ (18); and LD, the amount of which is known to be closely related to the size of the infarct (14). Because uric acid, creatinine, and urea, as well as CK and LD are highly correlated, we expected that little would be gained by adding the remaining variables.

Finally, the patient's status, "alive" or "deceased," was recorded every day for all patients in the data base.

\section{The Statistical Problem}

We are faced with the situation where patients are being measured at equally spaced time points $t$ (e.g., every day) up to a certain time $\mathrm{T}$, and their outcome recorded in every time interval $[t, t+1]$.

Let $y_{t}$ denote the outcome variable in the $t$ th interval of time, and assume that $y_{t}$ takes only two values, namely, 1 for "alive" and 2 for "dead." It follows that for each patient in the data base, we can define a "sequence of states" $\left(y_{1}, y_{2}\right.$, ...) of maximum length $T$. In our application, $T=7$ and every sequence is a series of 1 's terminated by either 1 or 2 depending on whether the patient survived or died. For example, a patient dying in the fourth time interval $[3,4]$ has the sequence $(1,1,1,2)$, because he was alive during the first three days after admission and died on the fourth. Likewise, a patient alive at the end of the one-week investigation period yields a full sequence of 1's.

Let $\boldsymbol{X}^{t}$ denote the vector of all observations made on the patient up to and including time $t$. This vector may consist of (a) baseline or "constant" variables measured only once, for example, age and previous MIs on admission, and (b) serial measurements of AGP, urea, and LD.

The question that we propose to answer-namely, how to assess on a daily basis the patient's survival chances from previously recorded measurements-reduces to the following statistical problem: "predict at each time-point $t$ the outcome variable $y_{t}$ from the information vector $\boldsymbol{X}^{t}$ available up to time $t$; or in other words, compute the probability $\operatorname{pr}\left(y_{t}=2\left(X^{t}\right)\right.$."

The problem is akin to classical regression analysis, where one proposes to predict the patient's outcome from a vector of observations. In the present situation, however, both the outcome and the predictor variables are time dependent. Additionally, there is a double selection problem, namely, (a) to determine the best combination of predictive variables, and $(b)$ to decide upon the number of past observations that are needed to achieve a satisfactory prediction at each time point. For instance, only the current or last two measurements of the selected variables might suffice for assessing the patient's risk, earlier observations having no effect on the outcome at time $t$.

Clearly, the number of possible "variable/time-point" combinations to be tested in the regression analysis increases dramatically with the number of components of vector $\boldsymbol{X}^{t}$. Thus, in practice, one usually proceeds hierarchically with models of increasing complexity and stops the process when no statistically significant improvement can be obtained in the prediction of outcome.

In sum, one must keep in mind the quest for the most parsimonious model and select the minimum number of past observations needed.

\section{The Prediction Model}

In the following, we shall assume that the vector $\boldsymbol{X}^{t}$ has the same length $\mathrm{p}$ at each time $t$, and denote its elements as $x_{1}, \ldots, x_{p}$, remembering that the values of these elements generally change with time $t$.

The basic model for prediction of a dichotomous response variable based on repeated measurements was recently proposed by Wu and Ware (19), and applied to three yearly measurements made in two cohorts obtained in the Framingham Heart Study. This model, namely,

$$
\operatorname{pr}\left(y_{t}=2 \mid X^{t}\right)=\exp \left(a_{0 t}+a_{t}^{\prime} X^{t}\right) /\left[1+\exp \left(a_{0 t}+a_{t}^{\prime} X^{t}\right)\right]
$$

assumes that the probability of death in the interval $[t, t+$ $1]$ is a logistic function of a linear combination of past observations (see also 20-22).

In the above equation $a_{t}^{\prime}=\left(a_{1 t}, \ldots, a_{p t}\right)$ is a vector of length $\mathrm{p}$ whose elements are generally time dependent. In vector notation, $a_{t}^{\prime} X^{t}$ means $a_{1} x+\ldots+a_{p} x p$. The complexity of model 1 will vary greatly depending on $(a)$ the number p of elements to be included in the observation vector $X^{t}$, and (b) whether the intercept $a_{0 t}$ or the vector of coefficients $a_{t}$ or both are assumed to be time dependent (if not, the subscript $t$ will be dropped).

The assumption of constant coefficients (stationary model) throughout the monitoring period, at least for the intercept $a_{0 t}$, is unrealistic in the acute-MI situation. Indeed, large variations are observed in the concentrations in serum of the biochemical markers investigated, especially during the first week after admission to the hospital. Conversely, if one assumes that the weighting factors $a_{i t},(i=0, \ldots, p)$, are time dependent (nonstationary model), the model conforms better to reality. The drawback, however, is that the weights have to be estimated for each variable at each time point-a difficult task. One possible solution would be to apply at each time $t$-i.e., every day-a classical discriminant analysis between survivors and "next-day" nonsurvivors. This approach is usually not feasible, because the number of deaths each day is small as compared with the number of patients still alive. Alternatively, one can subtract from the observations some deterministic part of the response to the disease process, so as to bring stability to the time series observed. In this case, a model with constant coefficients can be postulated for the transformed variables, greatly simplifying the computations.

The two-stage approach. Figures 1-3 display the average response curves for AGP, urea, and LD in three MI-patient categories of increasing risk, namely, $(a)$ the one-year survivors, (b) the patients who died between one and 12 months, and $(c)$ the deaths between seven days and one month. For each biochemical parameter, there was a clear ordering of the group response curves over the one-week 


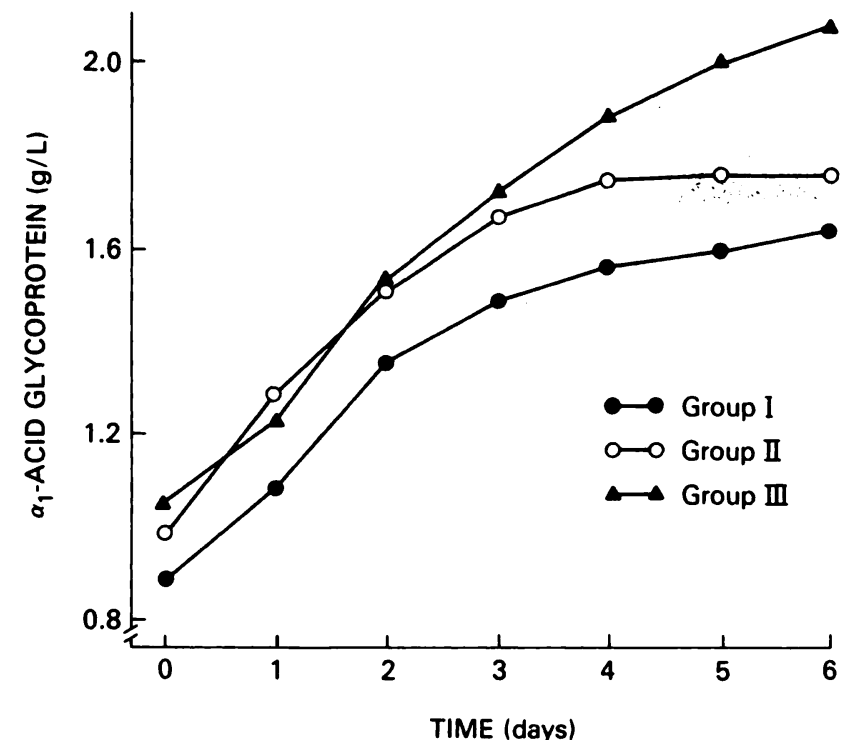

Fig. 1. Average response curves of serum $\alpha_{1}$-acid glycoprotein ( $\left.g / L\right)$ after acute $M I$ in one-year sunvivors (group I, $n=272$ ), deaths between one and 12 months (group II, $n=17$ ), and deaths between seven and 30 days (group III, $n=16$ ) after admission

Value of Zerbe's test for group differences is $F=7.05$, with degrees of freedom 2.77 and $418.77(p<0.001)$

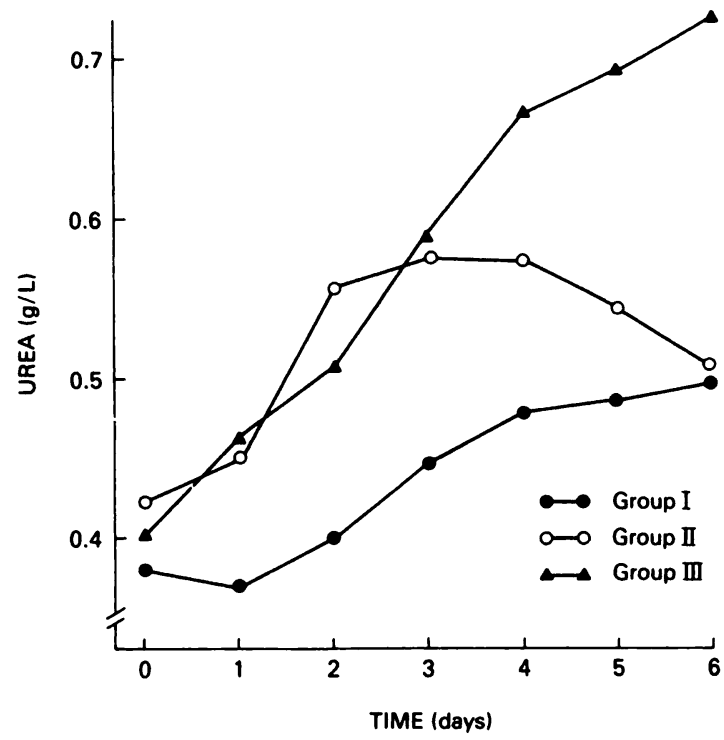

Fig. 2. Average response curves of blood urea $(g / L)$ after acute $\mathrm{MI}$ Value of Zerbe's test for group differences is $F=6.16$, with degrees of freedom 1.62 and $244.90(p<0.01)$. Groups as in Fig. 1

study period, confirming a correlation between in-hospital collected data and patient outcome after hospital discharge. We compared the average response curves, using Zerbe's nonparametric test (23), and found a statistically significant difference between the three groups (AGP: $F=7.05, p<$ 0.001; urea: $F=6.16, p<0.01$;D: $F=9.44, p<0.001$ ). From these results, one would expect that patients dying during the first week exhibit, on average, even steeper response curves than those found in the three groups above. For the purpose of our study, we regarded the average response curves of the one-year survivors (which represent more than $80 \%$ of our total patient population) as the typical evolutions of AGP, urea, and LD data after an MI, and any upper deviation from them was considered as an increase in the patient's risk. Hence we decided to correct every individual's response curves for the deterministic component by subtracting the curve representing the data for one-year

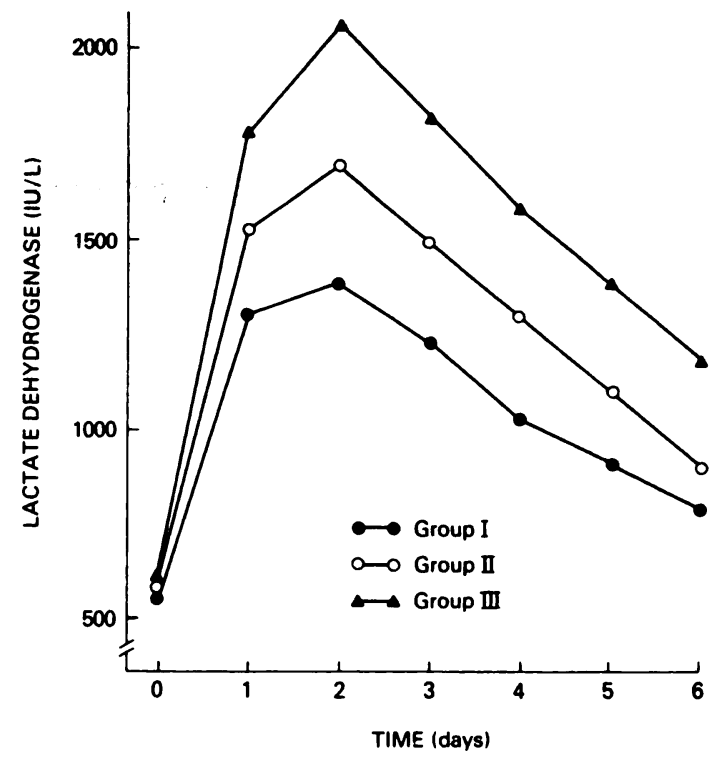

Fig. 3. Average response curves of lactate dehydrogenase (U/L) after acute MI

Value of Zerbe's test for group differences is $F=9.44$, with degrees of freedom 2.29 and $346.39(0<0.001)$. Groups as in Fig. 1

survivors. Because the response curves envisaged in our study were obtained by simple linear interpolation between successive measurements, the correction above merely consisted in standardizing every observation at time $t$, i.e., by subtracting the mean and dividing by the standard deviation for the one-year survivor group at that time. We emphasize that this transformation was applied only to time-dependent variables, not to baseline variables such as age or previous MIs.

Let $Z^{t}=\left(z_{1}, \ldots, z_{\mathrm{p}}\right)$ denote the vector of transformed elements of $X^{t}$. It is clear that, on average, the elements of $Z^{t}$ fluctuate around 0 for low-risk patients (one-year survivors) and around some positive value for high-risk patients (early deaths). Therefore the assumption of constant coefficients in the logistic model 1 becomes realistic.

In sum, we propose a two-stage approach for solving the problem of dynamic outcome prediction in acute myocardial infarction. The method consists of $(a)$ transforming the original time-dependent variables as described above, and (b) assuming for the transformed set of data the logistic model

$$
\operatorname{pr}\left(y_{t}=2 Z^{\prime}\right)=\exp \left(\mathrm{b}_{0}+b^{\prime} Z^{\prime}\right) /\left[1+\exp \left(\mathrm{b}_{0}+b^{\prime} Z^{\prime}\right)\right]
$$

where $b_{0}$ and $b^{\prime}=\left(b_{1}, \ldots, b_{p}\right)$ are now a set of unknown "constant" parameters to be estimated from the data base.

We shall call the linear function $S(t)=b_{0}+b^{\prime} Z^{t}$ the "risk index" at time $t$. As in classical discriminant analysis, this index can take any value on the real line, increased values being associated with high risk of death, easily seen from equation 2; conversely, large negative scores are signs of good prognosis.

Estimation. Model 2 can be postulated for any combination of vector $\boldsymbol{Z}$, depending on how many elements are included in this vector. For each model $\mathbf{M}_{\mathbf{i}}$ considered, the problem then reduces to estimating the coefficients of the risk function $S(t)$, by using the information contained in the data base. The number of parameters to be estimated for each model is obviously equal to $p+1$, i.e., the $p$ weights associated with each element of vector $\boldsymbol{Z}^{t}$ and the intercept $b_{0}$.

Estimation of the parameters was obtained by maximiz- 
ing the logarithm of the likelihood function of the sample, noted by $\log \mathrm{L}$.

To find out which model best fits the observations, we used a log-likelihood ratio criterion asymptotically distributed as a chi-square test. We also computed Akaike's Information Criterion (24), which is particularly appropriate when attempting to select a good model from among many alternatives. This criterion combines for each model the maximized likelihood (log $\hat{L}$ ) with the number of parameters estimated $(p+1)$. By definition, AIC $=-2 \log \mathrm{L}+$ $2(p+1)$, and the procedure chooses as the "best" model the one for which AIC is a minimum.

\section{Results}

For each patient in the data base, we associated a sequence of states as described above under The Statistical Problem. We tallied the number of nonsurvivors in each time interval, and included in the last interval all patients who died between days 7 and 15, patients with such early death also being considered as high-risk patients (Table 1). The total number who died during the monitoring period was 33 (10\%).

As described above, the time-dependent variables AGP, urea, and LD were standardized-i.e., $Z=(x-\bar{x}) / S D-$ to eliminate the deterministic component of the disease response and to apply the simplified model 2 . Table 2 gives the means and standard deviations of the response curves for the one-year survivors at each day for these three laboratory tests. We note from Table 2 and from Figures 1-3 that (a) there is a steady increase of AGP and urea from admission until the end of the first week, and (b) LD activities reach a maximum on the second day after admission, then regularly decrease until the last day.

\begin{tabular}{|c|c|c|c|c|c|}
\hline $\begin{array}{c}\text { Time } \\
\text { Interval, day }\end{array}$ & $\begin{array}{c}\text { State } \\
\text { sequence }\end{array}$ & $\begin{array}{l}\text { No. } \\
\text { deaths }\end{array}$ & $\begin{array}{l}\text { Cumulative } \\
\text { no. of } \\
\text { deaths }\end{array}$ & $\begin{array}{l}\text { No. of } \\
\text { survivors }\end{array}$ & $\begin{array}{l}\text { Total no. } \\
\text { of patients }\end{array}$ \\
\hline $\begin{array}{l}0-1 \\
1-2 \\
2-3 \\
3-4 \\
4-5 \\
5-6 \\
6-7\end{array}$ & $\begin{array}{l}2 \\
12 \\
112 \\
1112 \\
11112 \\
111112 \\
1111112\end{array}$ & $\begin{array}{l}3 \\
5 \\
8 \\
3 \\
2 \\
4 \\
8\end{array}$ & $\begin{array}{r}3 \\
8 \\
16 \\
19 \\
21 \\
25 \\
33\end{array}$ & $\begin{array}{l}327 \\
322 \\
314 \\
311 \\
309 \\
305 \\
297^{\circ}\end{array}$ & $\begin{array}{l}330 \\
327 \\
322 \\
314 \\
311 \\
309 \\
305\end{array}$ \\
\hline
\end{tabular}

- See text.

'The sequence of states associated with each of the 297 survivors at the end of the monitoring period is 1111111 .

Table 2. Daily Means and Standard Deviations for AGP, Urea, and LD for One-Year Survivors during the First Week after an MI^

\begin{tabular}{|c|c|c|c|c|c|c|}
\hline \multirow{2}{*}{$\begin{array}{l}\text { Day after } \\
\text { admission }\end{array}$} & \multicolumn{2}{|c|}{ AGP, g/L } & \multicolumn{2}{|c|}{ Urea, g/L } & \multicolumn{2}{|c|}{ LD, U/L } \\
\hline & Moan & SD & Moan & SD & Mean & SD \\
\hline $\begin{array}{l}0 \\
1 \\
2 \\
3 \\
4 \\
5 \\
6\end{array}$ & $\begin{array}{l}0.90 \\
1.07 \\
1.34 \\
1.50 \\
1.58 \\
1.60 \\
1.63\end{array}$ & $\begin{array}{l}0.21 \\
0.31 \\
0.28 \\
0.33 \\
0.38 \\
0.40 \\
0.43\end{array}$ & $\begin{array}{l}0.38 \\
0.37 \\
0.40 \\
0.45 \\
0.48 \\
0.49 \\
0.50\end{array}$ & $\begin{array}{l}0.13 \\
0.16 \\
0.18 \\
0.20 \\
0.21 \\
0.21 \\
0.23\end{array}$ & $\begin{array}{r}571 \\
1323 \\
1418 \\
1249 \\
1031 \\
916 \\
800\end{array}$ & $\begin{array}{l}292 \\
570 \\
623 \\
542 \\
412 \\
344 \\
291\end{array}$ \\
\hline
\end{tabular}

- These data are from 272 one-year survivors. The corresponding response curves are derived by linear interpolation between the mean values (see Figs. 1-3).

\section{Fitting of Models}

Several models were fitted to the data (Table 3). For each model $M_{i}$, we determined $(a)$ the maximum of the loglikelihood function, $\log \hat{\mathrm{L}} ;(b)$ the number of fitted parameters, p + 1; (c) Akaike's Information Criterion AIC, which enables one to select the best of a series of models; and $(d)$ the estimated coefficients $B_{0}, \ldots, b_{p}$ and their asymptotic standard errors. Finally a bias correction was applied to the estimated coefficients for the "best" model, according to a method by Anderson and Richardson (25).

Model M1: Prior probabilities. The simplest model is to assume that there are no covariates in the risk index, i.e., no baseline variables and no time-dependent variables; thus $S(t)=b_{0}$. This model is equivalent to assuming that, on the average, the prior probabilities of survival $\left(P_{1}\right)$ and death $\left(P_{2}\right)$ are constant in every time interval. The $\mathrm{ML}$ estimate of $b_{0}$ was found to be -4.18 . Consequently, by equation 2 , the daily probability of death is $\mathrm{P}_{2}=0.015$ and the probability of survival $P_{1}=0.985$. Allocation of the individual patients into survivor and nonsurvivor groups is not possible merely on the basis of overall prior probabilities. Therefore, it is anticipated that adding the baseline variables (age and previous MIs) and serial laboratory measurements into the risk function will improve predictions.

Model M2: Effect of age. The introduction of age significantly improved model $\mathrm{M} 1: \chi^{2}=2\left(\log \hat{\mathrm{L}}_{2}-\log \hat{\mathrm{L}}_{1}\right)=15.58$,

Table 3. Results from Fitting Various Models for Dynamic Outcome Prediction to the Data Base of 330 Patients with Acute MI

\begin{tabular}{|c|c|c|c|c|c|}
\hline Model:S(1) & $\begin{array}{l}\text { Estimated } \\
\text { coeff. }\end{array}$ & $\begin{array}{l}\text { SE of } \\
\text { coeff. }\end{array}$ & $\log C^{b}$ & $p+1$ & AIC \\
\hline $\begin{array}{l}\text { M1: Intercept } \\
\text { M2: Intercept } \\
\text { Age }\end{array}$ & $\begin{array}{r}-4.18 \\
-8.78 \\
.077\end{array}$ & $\begin{array}{l}.175 \\
1.30 \\
.020\end{array}$ & $\begin{array}{l}-171.55 \\
-163.76\end{array}$ & $\begin{array}{l}1 \\
2\end{array}$ & $\begin{array}{l}345.1 \\
331.5\end{array}$ \\
\hline $\begin{array}{l}\text { M3: Intercept } \\
\text { Age } \\
\text { PI }\end{array}$ & $\begin{array}{c}-8.94 \\
.076 \\
.51\end{array}$ & $\begin{array}{l}1.34 \\
.021 \\
.240\end{array}$ & -162.03 & 3 & 330.1 \\
\hline $\begin{array}{l}\text { M4: Intercept } \\
\text { Age } \\
\text { PI } \\
\text { AGP (t) }\end{array}$ & $\begin{array}{c}-8.92 \\
.074 \\
.50 \\
.26\end{array}$ & $\begin{array}{l}1.33 \\
.021 \\
.241 \\
.130\end{array}$ & -160.29 & 4 & 328.6 \\
\hline $\begin{array}{l}\text { M5: Intercept } \\
\text { Age } \\
\text { PI } \\
\text { Urea (t) }\end{array}$ & $\begin{array}{c}-7.85 \\
.059 \\
.66 \\
.48\end{array}$ & $\begin{array}{l}1.21 \\
.019 \\
.246 \\
.066\end{array}$ & -143.70 & 4 & 295.4 \\
\hline $\begin{array}{l}\text { M6: Intercept } \\
\text { Age } \\
\text { PI } \\
\text { LD (t) }\end{array}$ & $\begin{array}{c}-7.86 \\
.053 \\
.41 \\
.46\end{array}$ & $\begin{array}{l}1.32 \\
.021 \\
.27 \\
.077\end{array}$ & -143.39 & 4 & 294.8 \\
\hline $\begin{array}{l}\text { M7: Intercept } \\
\text { Age } \\
\text { PI } \\
\text { Urea }(t-1) \\
\text { Urea }(t)\end{array}$ & $\begin{array}{c}-7.91 \\
.050 \\
.65 \\
-.15^{a} \\
.58\end{array}$ & $\begin{array}{l}1.23 \\
.019 \\
.246 \\
.129 \\
.111\end{array}$ & -143.05 & 5 & 296.1 \\
\hline $\begin{array}{l}\text { M8: Intercept } \\
\text { Age } \\
\text { PI } \\
\text { LD }(t-1) \\
\text { LD (t) }\end{array}$ & $\begin{array}{c}-7.86 \\
.053 \\
.41 \\
.10^{a} \\
.41\end{array}$ & $\begin{array}{l}1.32 \\
.021 \\
.280 \\
.129 \\
.108\end{array}$ & -143.12 & 5 & 296.2 \\
\hline $\begin{array}{l}\text { M9: Intercept } \\
\text { Age } \\
\text { PI } \\
\text { Urea }(t) \\
\text { LD }(t)\end{array}$ & $\begin{array}{c}-7.14 \\
.038 \\
.58 \\
.38 \\
.31\end{array}$ & $\begin{array}{l}1.22 \\
.019 \\
.274 \\
.073 \\
.079\end{array}$ & -132.72 & 5 & $\begin{array}{c}275.4 \\
\text { (minimum) }\end{array}$ \\
\hline
\end{tabular}

- Nonsignificant at the $5 \%$ level by an asymptotically normal significance test.

'The chi-square test for comparing two models is obtained by taking twice the difference between the corresponding $\log \hat{L}$ values, and the number of degrees of freedom is equal to the difference between the number of parameters estimated in each model.

PI, no. of previous Mis. 
1 d.f. ( $p<0.001$ ). Akaike's criterion decreased by 13.6 units. The risk function obtained was $S(t)=-8.87+0.077$ age. Hence, a 70-year-old patient has, on the average, a daily probability of death of $P_{2}=0.033[S(t)=-3.39]$, twice as much as we found above.

Model M3: Effect of previous MIs. The addition of the number of previous MIs to age produced no significant difference at the $5 \%$ critical level ( $\chi^{2}=3.46$, not significant), although the ratio of $B_{2}$ to its standard error $(0.51 / 0.24=$ 2.13) exceeded 1.96, the upper $2.5 \%$ point of the gaussian distribution. We decided, however, to keep previous MIs in the risk index, because in previous studies $(6,22)$ this parameter was always significantly efficient in discriminating survivors and nonsurvivors.

Models M4-M6: Use of the current biochemical observation. In these models, we added to age and previous MIs only the most recent observation of AGP (M4), urea (M5), or LD (M6). By comparing each of these models with model M3, we found $L D$ to be the best biochemical parameter to use in improving outcome prediction $\left(\chi^{2}=37.28\right.$ vs 3.48 for AGP and 36.66 for urea), and concluded that AGP did not help much in assessing the current patient's condition. This result seems to contradict the high $F$-value (7.05) we found when comparing the three risk categories depicted in Figure 1. The two situations, however, are entirely different. In one case, we looked at early deaths only, and in the other at patients dying later, after hospital discharge. Actually, we showed that the effect of AGP only appears later in the course of the disease (15) and that AGP probably is the best biochemical test for making a long-term prognosis (26). Note that blood urea was nearly as good a predictor as LD. When we say this, what we mean is that the current (or most recent) value of $L D$ or urea at time $t$ is a good predictor of outcome in the next time interval.

At this point, we know that prediction is not significantly improved by AGP, but is improved by the knowledge of urea and LD. Now two further questions need to be answered: (a) Do past values of $L D$ or urea improve the predictive power of the current observation? (b) If we combine urea and LD, do we obtain a better risk index than using each test separately?

Models M7, 8: Effect of previous laboratory measurements. As the results clearly indicate, prediction was not significantly improved by adding to the current value (time $t$ ) the previous one (time $t-1$ ). For urea, the log-likelihood increased from -143.70 to -143.05 , leading to a $\chi^{2}$ value of 1.30 (not significant). Likewise for $\mathrm{LD}$, we found a nonsignificant value of $x^{2}=0.54$. Hence, only the most recent observation is needed to obtain satisfactory dynamic outcome prediction in patients suffering from MI. In fact, high correlations were found between successive standardized test results across the population.

Model M9: Joint effect of urea and $L D$. When we combined both urea and LD (most recent results) with age and previous MIs in the risk index, there was a considerable benefit in prediction efficiency. When we compared models M3 and M9, the log-likelihood ratio test obtained was highly significant: $\chi^{2}=58.64$ on two degrees of freedom. Model M9 was also the one for which the value for Akaike's Information Criterion reached a minimum, 275.44. Adding to M9 either AGP or previous observations of urea and $L D$ yielded only redundant and unnecessary information.

\section{The Dynamic Prognostic Index and Its Efficiency}

From the fitting of the various models, we concluded that the best risk index, that providing optimal dynamic outcome prediction, was given by the following equation (bias correction applied to the coefficients):
$S(t)=-7.14+0.038$ age $+0.58 \mathrm{PI}+0.38 \mathrm{u}_{t}+0.31 \mathrm{l}_{t}(3)$

where $\mathrm{PI}$ is the number of previous MIs, and $u_{t}$ and $l_{t}$ represent the current observations of urea and $L D$, standardized as described above (by using Table 2). Both of these standardized variables fluctuate around zero in one-year survivors, urea having slightly more weight $(0.38)$ than LD $(0.31)$ in assessing a patient's risk. Also note that because all the coefficients of the variables in equation 3 are positive, increased values of the variables are indicative of severity and bad prognosis.

To evaluate the overall efficiency of the dynamic prognostic index derived, we computed the daily values of the index (equation 3) for all 330 patients in the data base. For each of the 297 patients still alive at the end of the one-week monitoring period (survivors: group D), we determined the highest value of $S(t)$, i.e., the poorest score during the oneweek study period, whereas for the 33 patients who died (nonsurvivors: group D), we recorded the score just preceding death. We studied the distributions of these scores for both groups by applying a likelihood ratio approach as described elsewhere (27). By using the cutoff point where the two distributions intersect $\left(S^{*}=-3.88\right)$, we correctly allocated 238 survivors (specificity $=80 \%$ ) and 24 nonsurvivors (sensitivity $=73 \%$ ) to their groups, thus yielding a total efficiency of nearly $80 \%$. For comparative purposes, when the risk index was based on age and previous MIs alone (thus excluding the serial values of urea and $L D$ ), specificity and sensitivity dropped respectively to $67 \%$ and $58 \%$ (total efficiency, 66\%).

There is another way to appraise the predictive power of the risk index obtained. For each patient in the data base, we computed the accumulated probability of death conditional on the observations available from admission until the end of the seven-day period (or until death for nonsurvivors). If $P(t)$ denotes the conditional probability of death in the th interval of time as given by equation 2 , then the accumulated probability of death associated with every patient, noted $\pi$, is computed from the relation ${ }^{5}$

$\pi=\mathrm{P}(1)+\sum_{t=2}^{\tau} \mathrm{P}(t) \prod_{t^{\prime}=1}^{t-1}\left[1-\mathrm{P}\left(t^{\prime}\right)\right]$,

where $\tau$ equals the actual time of death for nonsurvivors and $(\tau=7)$ for survivors. As shown in Figure 4, the cumulative frequency distributions of these probabilities $\pi$ in survivors $(n=297)$ and in nonsurvivors $(n=33)$ are notably distinct. For instance, an accumulated probability of death of at least 0.15 was obtained in only $10 \%$ of survivors, but in more than $40 \%$ of nonsurvivors, emphasizing the difference between the two groups. Practically, one can say that any value of $\pi$ exceeding $10 \%$ was a clear sign of poor prognosis.

The excellent performance of this dynamic risk index derived from our data base was recently confirmed on a test sample of 76 more MI patients. From among the 11 patients who died during hospital stay, eight (73\%) had a score larger than the critical cutoff point of -3.88 before death. Among the 65 survivors, $54(83 \%)$ showed persistent negative values less than -3.88 during the one-week monitoring period.

\section{Interpretation of Individual Risk Scores}

The data above provide an overall evaluation of the

${ }^{5}$ If we regard the accumulated probability $\pi$ as a function of $\tau$ (= $2,3, \ldots)$, not as an end-study statistic, it could be computed sequentially (i.e., every day) as a warning sign that increased therapeutic effort is necessary. The complementary function $\sigma(\tau)=$ $1-\pi(\tau)$ actually represents the probability of survival conditional on past observations collected from the patient. 
efficiency of the dynamic prognostic index based on age, previous MIs, urea, and LD. We may, however, investigate the implication of a given index score on the predicted outcome of an individual patient. As an illustration (Table 4), we computed the daily risk scores $S(t)$ and the corresponding probabilities $P(t)$, using equations 2 and 3 , for two patients selected from the data base.

Patient 1 was 71 years old and had had one previous infarct. He showed increasing index scores from the time of admission, and he died on the third day. Before death $(t=2)$, he had a score of $S(2)=-1.36$ and his probability of death for the next day was 21\%. Comparing this result with the unconditional probability of death found previously (see model M1: $P_{2}=0.015$ ) shows more than a 10-fold increase.

Patient 2, 75 years old, died one month after hospitalization; he had had no previous infarct. His highest index score during the first week of hospital stay was recorded on day 4, $S(4)=-2.30$. This value, well above the critical score of -3.88 , indicates that the patient had an index value that is found more frequently in nonsurvivors than in survivors. Likewise his probability of death at that time was 0.091 , sixfold greater than the prior probability of 0.015 . These results confirm that the patient was already at high risk during his hospital stay, and they may have provided a hint of the late fatal outcome.

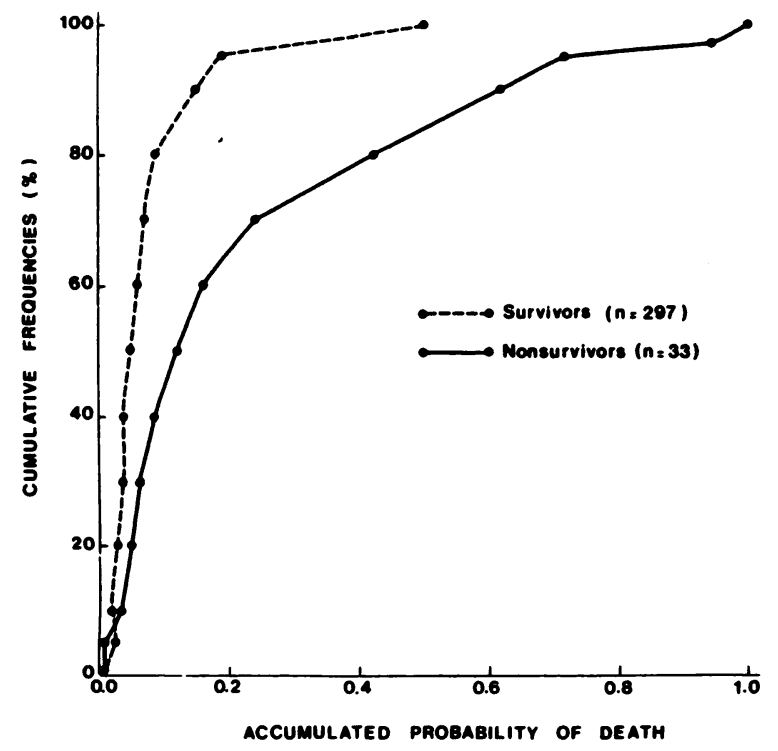

Fig. 4. Cumulative frequency distributions of the accumulated conditional probability of death $(\pi)$ in survivor $(n=297)$ and nonsurvivor $(n=$ 33) groups
The practical use of the methodology proposed is straightforward. For any new patient entering the coronary-care unit, record the age and number of previous infarctions. Each time results for urea and LD become available, standardize these results and compute the value $\mathbf{S}(t)$, using equation 3; finally, equation 2 provides an estimated probability of death (and of survival). The risk index $\mathrm{S}(t)$ can be considered as summarizing the joint value of age, previous MIs, and biochemical information at time $t$. Instead of having to interpret several results, the clinician is presented with a single score, which we know is optimal with regard to the patient's past and present history for predicting immediate future outcome. Obviously, the clinician is receiving additional pieces of information from other sources, so the value of $S(t)$ has to be integrated into the general clinical assessment of the patient's risk.

\section{Discussion}

Serial measurements are a natural part of laboratory medicine, e.g., in health surveillance, therapeutic monitoring, and intensive care.

Recently, there has been growing concern as to the best use of such cumulative results for prognostic and predictive purposes $(1,6,28)$. For example, Harris $(29,30)$ has provided a comprehensive methodology for monitoring individuals enrolled in a health-surveillance plan and sampled at regular time intervals. Models, such as the strictly homeostatic (assuming independent serial observations) or the nonstationary random walk, may also be indicated for detecting changes in stable pathophysiological processes. Until now, however, their use in patients has been quite limited and may have been hindered by interfering factors such as drug administration and treatment. Recently, Winkel et al. (31) considered the problem of predicting, from cumulative laboratory results, recurrence in patients with breast cancer and found that an appropriate statistical treatment of such results considerably improved the prediction of recurrence. All the potential benefits of these pioneering studies may not yet have been fully perceived, but clearly in many clinical situations serial laboratory measurements are improperly used and underevaluated.

In acute diseases, as opposed to stable disease processes, biochemical components do not always fluctuate around some threshold value but oftentimes follow a typical deterministic pattern. As we mentioned before, after an acute MI, there is a characteristic rise and fall in serum enzymes such as creatine kinase, its isoenzyme CK-MB, $\mathrm{LD}$, and aspartate aminotransferase; serum urea and AGP concentrations tend to increase (15).

In this paper we have investigated the problem of dynam-

\section{Table 4. Dynamic Risk Scores S(t) and Associated Probabilities of Death P(t) Calculated for Two}

Patients with Acute MI

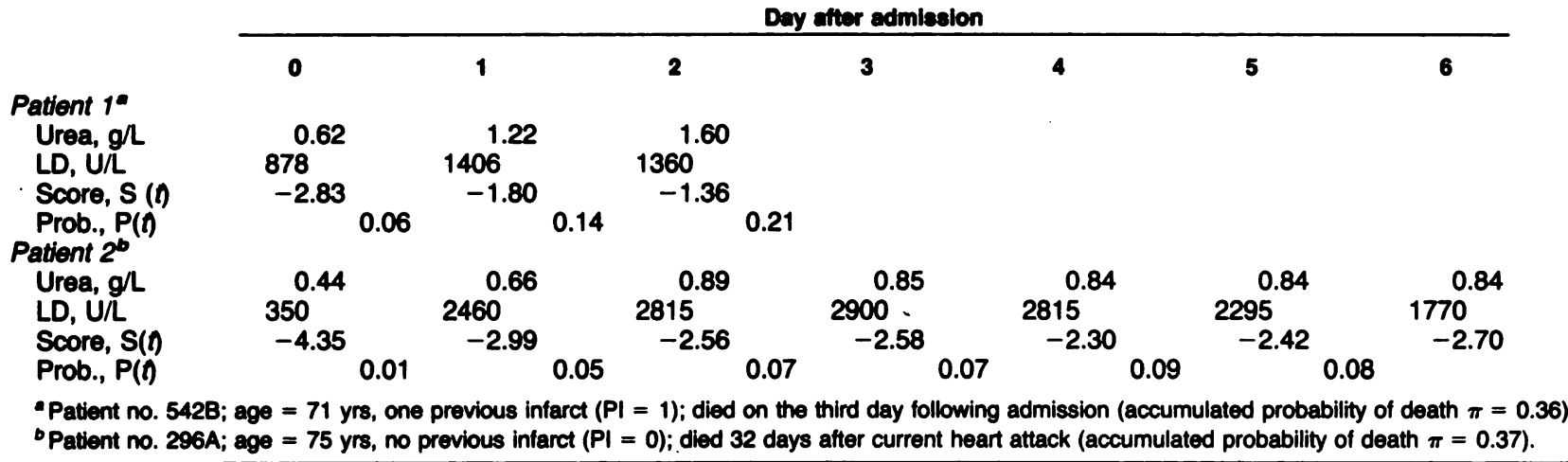


ic outcome prediction, in terms of death or survival, from laboratory data recorded serially during the patient's stay in the coronary-care unit. Most methods suggested in the literature to date $(4,22)$ are too rigid, in the sense that they do not emphasize the dynamics of the disease process, but instead base prediction on a single multivariate observation. The vector may, for example, consist of data available on admission and of the maximum enzyme values recorded during hospitalization. By proceeding this way, patients dying before enzyme values reach their maximum are discarded from the study.

The approach we developed overcomes these drawbacks and makes optimal use of the outcome (or state sequence) and serial data of every patient in the data base. We assume that measurements are made at regular time intervals_for instance, every day - and that there is a logistic relation between survival probability and risk index. It is known, however, that logistic models are robust and applicable to a mixture of discrete and continuous variables $(20,21,27)$. Moreover, as shown in our application, baseline variables (e.g., age, previous MIs) and time-dependent variables (e.g., urea, LD) can be handled with equal facility.

We have also emphasized that maximum likelihood estimation of the coefficients of the linear risk index is simplified when these coefficients do not depend on time (stationary model). To achieve this property in our series of observations, we adjusted the individual response curves by subtracting the deterministic component of the evolution. We decided to consider the one-year survivors (patients at lowest risk in our data base) as providing the typical evolutionary pattern of $L D$ and urea after MI. The choice of the deterministic component should not affect the general results of our study, for we are interested in outcome prediction in the early phase of the disease. It is merely a convenient way of stabilizing the observation series and allowing the use of a simple logistic regression model.

Test selection considerations in dynamic prognosis have been alluded to and are twofold. First, which biochemical test should be included in the dynamic index, and what combination of tests provides the best prediction? Second, how many past observations should be used for each variable? In our application, we studied three constituents but only urea and LD were useful discriminators; AGP did not contribute significantly to outcome prediction. Furthermore, the most recent observations of urea and $L D$ were sufficient for assessing satisfactorily the patient's status.

Our results confirm the importance of $L D$ in short-term prognosis of acute $\mathrm{MI}$ and its relationship to infarct size (14). They also re-emphasize the preponderant role of serum urea in monitoring patients with acute diseases (18). As for age and number of previous episodes of MI, several studies have confirmed their prognostic effectiveness in acute $\mathrm{MI}(6,22)$.

In conclusion, the linear risk index derived from our data base enables one to predict, on a daily basis, the patient's survival chances from simple demographic data and from urea and LD measurements, as they become available. We believe that the methodology developed should enhance the interpretation of cumulative laboratory results, not only in acute MI, but also in any disease in which there is an acute phase during which the patient is closely monitored and multiple serial measurements are made.

This research project was carried out while A.A. was a Fogarty International Research Fellow (grant F05 TW02964) at the Laboratory of Applied Studies, National Institutes of Health, Bethesda, MD.

\section{References}

1. Groth $\mathrm{T}$, de Verdier $\mathrm{CH}$. Biodynamic models as pre-processors of clinical laboratory data. In Advanced Interpretation of Clinical Laboratory Data, C Heusghem, A Albert, ES Benson, Eds., Dekker, New York, NY, 1982, pp 151-170.

2. Werner M, Brooks SH, Mohsbacher RJ, Wasserman AG. Diagnostic performance of enzymes in the discrimination of myocardial infarction. Clin Chem 28, 1277-1302 (1982).

3. Solberg HE. Discriminant analysis. Crit Rev Clin Lab Sci 9, 209-242 (1978).

4. Albert A. Statistical methodology of prognostic indices derived from measurements made during hospital stay for acute myocardial infarction. In The First Year after a Myocardial Infarction, HE Kulbertus, HJJ Wellens, Eds., Futura Publishers, Mount Kisco, NY, 1983. In press.

5. Afif AA, Sacks ST, Liu VY, et al. Accumulative prognostic index for patients with barbiturate, glutethimide and meprobamate intoxication. N Engl J Med 285, 1497-1502 (1971).

6. Albert A, Chapelle JP, Heusghem C, et al. Evaluation of risk using serial laboratory data in acute myocardial infarction. In ref. 1 , pp 117-130.

7. Albert A. Discriminant analysis based on multivariate response curves: A descriptive approach to dynamic allocation. Stat Med 2 , 95-106 (1983).

8. Rosalki SB. An improved procedure for serum creatine phosphokinase determination. J Lab Clin Med 69, 696-705 (1967).

9. Wroblewski F, La Due JS. Lactate dehydrogenase activity in blood. Proc Soc Exp Biol Med 90, 210-213 (1955).

10. Mancini G, Vaerman JP, Carbonara AO, Hermans JF. Immunochemical quantitation of antigens by radial immunodiffusion. Immunochemistry 2, 235-254 (1965).

11. Marsh WH, Fingerhut $B$, Miller $H$. Automated and manual direct methods for the determination of blood urea. Clin Chem 11, 624-627 (1965).

12. Chasson AL, Grady HJ, Stanley MA. Determination of creatinine by means of automatic chemical analysis. Am J Clin Pathol 35, 83-88 (1961).

13. Sobrinho-Simoes MA. A sensitive method for the measurement of serum uric acid using hydroxylamine. J Lab Clin Med 65, 665668 (1965).

14. Chapelle JP, Albert A, Heusghem $C$, et al. Predictive value of serum enzyme determinations in acute myocardial infarction. Clin Chim Acta 106, 29-38 (1980).

15. Chapelle JP, Albert A, Smeets JP, et al. The prognostic significance of serum $\alpha_{1}$-acid glycoprotein changes in acute myocardial infarction. Clin Chim Acta 115, 199-209 (1981).

16. Chapelle JP, Albert A, Boland J, et al. Critical evaluation of serum uric acid levels in acute myocardial infarction. Clin Chim Acta 121, 147-157 (1982).

17. Agostini A, Binaghi PC, Radice F, Stabilini R. Acute phase proteins and healing of myocardial infarction. $J$ Mol Cell Cardiol 4 , 519-524 (1972).

18. Bigger JJ, Heller CA, Wenger TL, Weld FM. Risk stratification after acute myocardial infarction. Am J Cardiol 42, 202-210 (1978).

19. Wu MC, Ware JH. On the use of repeated measurements in regression analysis with dichotomous responses. Biometrics 35 , 513-521 (1979).

20. Walker SH, Duncan DB. Estimation of the probability of an event as a function of several independent variables. Biometrika 54, 167-179 (1967)

21. Anderson JA. Separate sample logistic discrimination. Biometrika 59, 19-35 (1972).

22. Chapelle JP, Albert A, Smeets JP, et al. Early assessment of risk in patients with acute myocardial infarction. Eur Heart $J 2$, 187-196 (1981)

23. Zerbe GO. Randomization analysis of the completely randomized design extended to growth and response curves. J Am Stat Assoc 74, 215-221 (1979).

24. Akaike $H$. Use of an information theoretic quantity for statistical model identification. Proc. Fifth Hawaii Int. Conf. on System Sciences, Western Periodicals Co., North Hollywood, CA, 1972, pp 249-250.

25. Anderson JA, Richardson S. Logistic discrimination and bias 
correction in maximum likelihood estimation. Technometrics 31, 71-78 (1979).

26. Chapelle JP, Albert A, Kulbertus HE, Heusghem C. The longterm prognostic significance of biochemical measurements obtained during the acute phase of myocardial infarction. In ref. 4.

27. Albert A. On the use and computation of likelihood ratios in clinical chemistry. Clin Chem 28, 1113-1119 (1982).

28. Bourguignat A, Férard G, Jung G, et al. Multivariate analysis of plasma enzyme profiles in severe head injury. Clin Chem 29, 107-109 (1983).
29. Harris EK. Some theory of reference values. II. Comparison of some statistical models on intra-individual variation in blood constituents. Clin Chem 22, 1343-1350 (1976).

30. Harris EK, Cooil BK, Shakarji G, Williams GZ. On the use of statistical models of within-person variation in long-term studies of healthy individuals. Clin Chem 26, 383-391 (1980).

31. Winkel P, Bentzon MW, Statland BE, et al. Predicting recurrence in patients with breast cancer from cumulative laboratory results: A new technique for the application of time series analysis. Clin Chem 28, 2057-2067 (1982). 